\title{
Features of teaching swimming to children of primary school age with autism spectrum disorder
}

\author{
E. Sh. Shayakhmetova ${ }^{*}$, L. M. Matveeva ${ }^{2}, A$. A. Sitdikova ${ }^{1}$, and V. A. Sokolova ${ }^{1}$ \\ ${ }^{1}$ Volga State Academy of Physical Culture, Sports and Tourism, Kazan, Russia \\ ${ }^{2}$ Bashkir State Pedagogical University named after M. Akmulla, Ufa, Russia
}

\begin{abstract}
The article presents an analysis of the peculiarities of teaching swimming to children of primary school age with autism spectrum disorder. The study was conducted on the basis of the sports complex "Burevestnik" in Kazan. According to the results of testing on the scale of assessment of the degree of autism of children, a group of boys aged 9-10 years was formed. In four children, the degree of autism is not expressed and moderately pronounced, in one child-a severe form of autism. At the time of the examination, the children did not know how to swim. Classes on the water were held for six months 3 times a week for 40 minutes. The children trained using the author's equipment - special boards with an associative character (a fish with bubbles, a whale with a fountain, a dolphin, a frog, a turtle, a rocket). The size of the swimming board remained standard, $32 \times 25 \mathrm{~cm}$. The development of the supporting device was due to the need to eliminate the inadequate response to various sensory stimuli, such as touching strangers or tactile contact with unfamiliar objects and textures, as well as to obtain positive associations and mental images for the successful development of a new exercise. At the end of six months of classes, all children with autism spectrum disorder have mastered swimming training according to the indicators: 1) immersion in water with exhalation in water, 2) sliding on the chest with the movement of the legs, 3) sliding on the chest with the movement of the legs and performing proper breathing. The study was determined by the ability to use boards to swim copyright form associative nature.
\end{abstract}

\section{Introduction}

A disorder that occurs as a result of impaired brain development and is characterized by a pronounced and comprehensive lack of social interaction and communication, as well as limited interests and repetitive actions, is considered to be an autism spectrum disorder (ASD) [5]. Brain disorder leads to communication difficulties and obsessions, but does not affect intelligence and learning ability [11].

Swimming classes play an important role in improving the quality of life and activity of a child with autism. Classes in the pool help to restore the disturbed or limited motor

* Corresponding author: Shaga.elv@yandex.ru 
functions of the child's body $[2,4,9]$. In swimming classes, a child with an autism spectrum disorder can learn to consciously control their body, develop muscle strength, agility, and improve their sense of balance and coordination of movements $[3,6]$.

To date, the autism spectrum disorder remains poorly understood [7, 8, 10], which does not allow us to properly consider the variety of means and methods of teaching swimming to children with this disease. As a rule, trainers use the same initial training for children with autism as for conditionally healthy children [1]. This fact predetermined the present study.

The aim of the study is to study the peculiarities of teaching swimming to children of primary school age with autism spectrum disorder.

\section{Materials and methods}

The study was conducted on the basis of the sports complex "Burevestnik" in Kazan. According to the test results on a scale measure the degree of autism for "Asperger's" was formed by a group of boys 9-10 years. In four children, the degree of autism is not expressed and moderately pronounced, in one child-a severe form of autism. At the time of the examination, the children did not know how to swim. Classes on the water were held for six months 3 times a week for 40 minutes.

Swimming fitness was evaluated according to the indicators proposed by T. I. Osokina: 1) immersion in water with exhalation in water, 2) sliding on the chest with the movement of the legs, 3) sliding on the chest with the movement of the legs and performing proper breathing (25-meter segment without stopping).

Mathematical and statistical processing of the obtained material was carried out on a personal computer using the Attestat software package using generally accepted methods of variation statistics.

\section{Results and discussion}

It is known that children with ASD have an inadequate strong reaction to sensory stimuli, such as tactile contact with unfamiliar objects or textures $[9,12]$. Therefore, the inventory that is used in the classroom (its shape, color and size) is of great importance. In this regard, the swimming classes used the boards developed by us with an associative character: a fish with bubbles, a whale with a fountain, a dolphin, a frog, a turtle, a rocket (the size of the board for swimming remained standard, $32 \times 25 \mathrm{~cm}$ ). For example, the "Bubble Fish" swimming board was used to teach proper breathing (inhaling through the mouth, exhaling through the nose). The child lowered his face into the water and exhaled, i.e. created bubbles like in the drawing of a fish.

Swimming fitness was evaluated twice, at the beginning and end of the study. To perform the control exercise, children with ASD were given one attempt, as practice has shown that the results of subsequent attempts are usually worse. The second attempt was granted only if the first attempt failed. The results are presented in table 1. 
Table 1. Swimming fitness of primary school-age children with autism spectrum disorder in the study.

\begin{tabular}{|l|c|c|c|c|}
\hline \multirow{2}{*}{ Swimming level } & \multicolumn{2}{|c|}{$\begin{array}{c}\text { Before the study } \\
(\mathbf{n = 5})\end{array}$} & \multicolumn{2}{c|}{$\begin{array}{c}\text { After the study } \\
(\mathbf{n = 5})\end{array}$} \\
\cline { 2 - 5 } & Quantity & \% & Quantity & \% \\
\hline Low (1-3 points) & 5 & 100 & - & - \\
\hline Average (4-7 points) & - & - & 4 & 80 \\
\hline High (8-12 points) & - & - & 1 & 20 \\
\hline
\end{tabular}

At the beginning of the experiment, the children with ASD did not know how to swim and, accordingly, no child coped with the control task. At the end of six months of classes, all children with ASD have mastered swimming training according to the indicators: 1) immersion in water with exhalation in water, 2) sliding on the chest with the movement of the legs, 3) sliding on the chest with the movement of the legs and performing proper breathing. In accordance with the rating scale for T. I. Osokina's children with ASD demonstrated an average level of swimming fitness, their average group indicator was $5.4+0.4$, and the differences in the indicators before and after the experiment reached statistically significant differences.

A post-experimental survey of parents of children with autism spectrum disorder revealed that the children became less ill, the children's muscular corsets became much stronger, they did not have sluggish movements, the children looked more coordinated and had much better control of their bodies.

\section{Conclusion}

In swimming, one of the most common supporting devices is a board. From the first lesson, the coach uses swimming boards, which usually have a traditional shape and color from the manufacturer. But, when teaching swimming to children with ASD, it is necessary to achieve positive associations and mental images for the successful development of a new exercise. Therefore, when teaching swimming to children with autism spectrum disorder, it is recommended to use boards with an associative character.

\section{References}

1. Ilyin V. A. Features of teaching swimming to children with autism / V. A. Ilyin, A.V. Ilyin // Modern aspects of physical culture, sports and psychological and pedagogical work with students: materials of the correspondence scientific and practical conference with international participation. - Penza State University-2017. - pp. 57-60.

2. Martynova O. G. Graphic justification of adaptive physical culture as a technology of rehabilitation of children with autistic spectrum disorders / O. G. Martynova, E. H. Valishin / / Young scientist. - 2017. - No. 4. - p. 337-342.

3. Udovichenko I. A. Health-improving swimming for children of primary school age with autism / I. A. Udovichenko, E. G. Kokoreva / / Physical culture and sport: science, education, technologies: materials of the All-Russian scientific and practical conference of undergraduates. - Ural State University of Physical Culture. - 2019. - p. 378-381.

4. Fomicheva E. N. The possibilities of swimming in the rehabilitation of school-age children with autistic spectrum disorder / E. N. Fomicheva, K. D. Valyaev / / National Association of Scientists. - 2015. - No. 6. - pp. 66-69. 
5. Tsekalo G. A. Psychological features of children with early childhood autism / G. A. Tsekalo, D. V. Turtseva // Proceedings of young scientists of the Altai State University. -2015. - No. 10. - pp. 386-387.

6. Chernomor A.V. Positive influence of classes in the pool for children with ASD / A.V. Chernomor / / Physical education and sport in higher educational institutions: a collection of articles of the XV International Conference dedicated to the 65th anniversary of BSTU named after V. G. Shukhov. - Belgorod State National Research University. - 2019-p. 204-207.

7. Art Dowdy, Matt Tincani (2019) Assessment and treatment of high-risk challenging behavior of adolescents with autism in an aquatic setting. Journal of Applied Behavior Analysis 45.

8. Andrew M. Colombo-Dougovito, Martin E. Block (2019) Fundamental Motor Skill Interventions for Children and Adolescents on the Autism Spectrum: a Literature Review. Review Journal of Autism and Developmental Disorders 6:2, pp. 159-171.

9. Nason V. (2016) Core Challenges in Autism. Sensory aspects of autism. Continuation // Autism and Developmental Disorders (Russia). Vol. 14. №. 3 (52). pp. 42-48. doi:10.17759/autdd.2016140304

10. Mohamed S. E. (2017) Effect of Aquatic Exercises Approach (Halliwick-Therapy) on Motor Skills for Children with Autism Spectrum Disorders //Ovidius University Annals, Series Physical Education and Sport Science, Movement and Health. Vol. 17. №. 2. pp. 20-27.

11. Fred R. Volkmar, Brian Reichow, James C. McPartlan (2016) Adolescents and Adults with Autism Spectrum Disorders. Springer. pp. 337.

12. Righi G. et al. Predictors of inpatient psychiatric hospitalization for children and adolescents with autism spectrum disorder / / Journal of autism and developmental disorders. - 2018. - Vol. 48. - no. 11. - pp. 3647-3657. 